\title{
X-RAY METHOOS FOR THE CHEMICAL CHARACTERIZATION OF ATMOSPHERIC AEROSOLS
}

\author{
J.M. Jak levic and A. C. Thompson \\ Lawrence Berkeley Laboratory \\ University of California \\ Berkeley, California 94720 U.S.A.
}

May 1981

This work was supported in part by the U.S. Environmental Protection Agency under interagency agreement number IAG AD-89- $\mathrm{F}-0-013-0$ with the Assistant Secretary for Environment, Office of Heal th and Environmental Research. Pollutant Characterization and Safety Reseerch Division of the U.S. Department of Energy under Contract W-7405-ENG-48. 


\title{
$X$-RAY METHODS FOR THE CHEMICAL CHARACTERIZATION OF ATMOSPHERIC AEROSOLS
}

\author{
J.M. Jaklevic and A.C. Thompson \\ Lawrence Berkeley Laboratory \\ University of California \\ Berkeley, California 94720 U.S.A.
}

We describe the development and use of several X-ray methods for the chemical characterization of atmospherical aerosol particulate samples. These methods are based on the emission, absorption and scattering of $X$-ray photons with emphasis on the optimization for the non-destructive analysis of dilute specimens. Techniques discussef include photon induced energy dispersive $x$-ray fluorescence, extended $x$-ray absorption fine structure spectroscopy using synchrotron radiation and high-rate $X$-ray powder diffractometry using a position-sensitive gas proportional counter. We have applied these $x$-ray analysis methons to the measurement of the chemical compositions of sizesegregated aerosol particulate samples obtained with dichotomous samplers. The advantages of the various methods for use in such measurements are described and results are presented. In many cases, the complementary nature of the analytical information obtained from the various measurements is an important factor in the characterization of the sample. For example, the multiple elemental analyses obtained from $X$-ray fluorescence can be used as a cross check on the major compounds observed by powder diffraction. 
1. Introduction

Over the past several years, a number of physical methods of analysis have been applied to the chemical characterization of environmental samples. Particularly in the case of atmospheric particulate samples, there has been extensive development work in the application of neutron activation analysis and X-ray fluorescence analysis using both charged particle and $x$-ray excitation[1,2]. In parallel with these developments, there have been improvements in the technology of aerosol samp!ing. The typical sample now consists of microgram quantities of size-segregated particles which are deposited on low mass substrates and which have been collected from the atmosphere in a precisely controlled fashion $[3,4]$. The idea? analytical method must then be able to perform accurate, sensitive, and non-destructive analysis of these minute quantities.

Because of the ease with which $x$-ray photons $c$ an be generated in the laboratory and berause of their negligible damaging effects on typicai samples, methods using $x$-rays as the excitation source have been widely used. We describe the use of three such methods for the elemental and chemical characterization of atmospheric aeroso? samples. The methods are eriergy dispersive $x$-ray fluorescence analysis, x-ray absorption pectroscopy and x-ray powder diffractometry. In each case the application of modern $x$-ray source and detector technology has greatly enhanced the potential of the method.

\section{X-Ray Fluorescence Elemental Analys is}

The application of energy dispersive $x$-ray fluorescence analys is using $S i(L i)$ semiconductor detector spectrometers to the problem of atmospheric 
aerosols has been widely jiscussed in the literature[1,4,5]. Fluorescence excitation of the sample can be provided either by photon sources or charged particle beams. Wa have employed pulsed $x$-ray tube excitation with secondary targets in order to perform elemental analysis of a broad range of elements with near optimal sensitivity. Table 1 is a list of elements which are routinely measured in our aerosol analysis program together with the measured sensitivities. Comparison of these values with ambient leveis of these elements in the atmosphere show that the method is more than adequate for routine measurements. To cover the entire range of elements, three separate spectra are accumulated using three secondary targets requiring a total measurenent time of five minutes. The data acquisition and reduction are totally automated and the results are stored and sorted in a large disk file. Over the past five years, a tatal of approximate $7 y$ 40,000 samples have been analyzed in this fashion[6].

The ability to perform multiple-element analyses for large sample sets results in a data resou.ce which can be used in many ways. Concentrations of individual elements can be used as monitors of specific pollutant sources within the region. Two examples might be sulfur as an indicator of fossil fuei power plants and lead or bromine as tracers for automotive activity[7]. In studies where multiple sampling sites are involved, it is possible to correlate elemental distributions with meteorological data in order to pinpoint discrete sources[8]. The availability of large data sets also facilitates the use of sophisticated statistical techniques for the discovery of common factors which are associated with certain elemental groups[9]. Finally, the elemental data often pinpoint specif ic samples for further analysis by other methods such as those discussed below. 


\section{Chemical Analysis}

Some of the more advantageous features of elemental analys is using $x$-ray fluorescence are the stability of the calibration and the inmunity of the results from chemical interferences due to unpredictable interactions with other elements. These features can be largely attributed to the relative isolation of the inner shell atomic electrons from any chemical effects involving the outer shells. Indeed, attempts to apply high resolution x-ray emmission spectroscopy for the measurement of chemical shifts have met with little success due to the small magnitude of the effects involved[10]. For this reason, we have explored the use of alternative $x$-ray methods for the characterization of chemical forms of the element of interest.

\section{X-Ray Absorption Spectroscopy}

Although the binding energies of inner-shell electrons are affected by the valence state of the atom, very little shift is noted in the emission spectra of the fluorescence $x$-rays. This results from the nearly equal energy shifts which occur in both the initial and final state orbital. However, methods which measure the level energy directly can exhibit shifts on the order of $10 \mathrm{eV}$ over a range of valence states. This is greater than the line widths of the levels involved and well within the energy resolution capabilities of electron detection methods such as Auger and photoelectron spectroscopy.

It is also possible, in principal, to indirectly measure the binding energy shift as a function of valence state by observing the abrupt increase in the photoelectric ionization crcss section when a narrow energy 
resolution photon beam is scanned in energy across the absorption edge of the inner shell. In practice, one observes a more complicated absorption spectrum which exhibits considerable structure in the region near the major absorption edge. This structure is likewise sensitive to the chemical state of the element and $c$ an be used to supplement the energy shift data. The ability of $x$-ray absorption spectra to exhibit chemical effects has been known for many years. However, the application of the method as an analytical tool for the characterization of dilute specimens has awaited the recent availability of high-energy, high-intensity synchrotron radiation sources. We have measured the absorption spectra of a number of elements present in atmospheric aerosol particulate samples using the variable energy $x-r a y$ beam at the Stanford Synchrotron Radiation Laboratory (SSRL).

Figure $I$ is a schematic diagram showing the experimental procedure used in the measurement[11]. The scanning $x-r a y$ beam is derived from the synchrotron radiation continuum using a double crystal monochromator. For the energy ranges used for the stufy, typical x-ray fluxes were approximately $10^{9}$ photons/sec in a less than $2 \mathrm{eV}$ energy window. This finely collimated beam (approximately $1 \mathrm{~cm} \times 1 \mathrm{~mm}$ ) was then incident on the sample and the energy dependence of both the absorption and fluorescence produced by the particular element in the sample was measured. In cases where the total absorption is small, the fluorescence signal is the more sensitive indicator of the absorption edge structure. For most of the elements analyzed in the present study, the concentrations were in the range of a few $\mu \mathrm{g} / \mathrm{cm}^{2}$. In order to enhance the sensitivity, $x-r a y$ fluorescence peak intensities obtained from three closely spaced semiconductor detector spectrometers were summed to give the final cross sections. 
The measurentent consists of tuning the crystal monochromator to an energy near the absorption edge of interest and then stepping the energy of the beam across the absorption edge. It should be noted, that because of the 1 to $2 \mathrm{eV}$ energy resolution of the beam as compared to the several hundred $\mathrm{eV}$ separation between the major absorption edges of the element of interest, very little concern need be given to interferences from other elements in the sample.

Figure 2 shows a selection of spectra obtained from three standard $2 n$ compounds which are of interest in air pollution studies. The interpretation of the observed structure requires two explanations. For incident photon energies below the absorption edge energy, it is possible to absorb a fhoton by promoting an electron to a bound but unoccupied state near the continum distribution. This gives rise to the series of sharp lines which occur at the beginning of the spectrum. Above the absorption edge energy, there are modulations in the cross section which are the result of resonance effects in the outgoing photoelectron waye which are caused by the spatial relationship of nearest neighbor atoms. This extended $x-$ ray finestructure (EXAFS) can persist for several hundred $\mathrm{eV}$ above the edge and is most evident in the ZnO spectrum.

Obviously, both types of fine structure are influenced by the chemical form of the element. In principal it is possible to perform a detailed theoretical analysis of the spectra and interpret them in terms of valence effects and crysta\}lography[12]. However we have taken the simpler approach of referring our unknown spectra to a series of standard spectra which constitute the most probable interpretations. These probable 
interpretations are influenced strongly by the observed elemental concentrations which had been obtained by prior $x-r a y$ fluorescence analysis.

Figure 3 shows the case of absorption spectra obtained on two size fractions acquired with a dichotomous sampler at a sampling site in urban St. Louis. The important feature to note is the obvious difference in chemical form between the coarse ( $2.4 \mu \mathrm{m}$ ciameter) and fine $(<2.4 \mathrm{\mu m}$ diameter) particles. Comparison with the spectra of Fig. 2 leads one to conclude that the fine particles are either zinc sulfate or zinc ammonium sulfate wilereas the coarse particles are predominantly zinc oxide. Since the samples were collected from the same volume of ambient air, this difference reflects the distinct chemical origin and subsequent transformation properties of the coarse and fine particles in the urban atmosphere.

A though the elaborate methodology required for $x$-ray absorption spectroscopy may seem unwarranted for the routine analysis of air pollution samples, it possesses several unique advantages which argue for its use in selected cases. The ability to analyze specific elements and determine their chemical form is in many ways unique. Compared to other physical methods such as various electron spectroscopies, the $x$-ray absorption method has the property of analyzing the bulk of the individual particles as opposed to being limited to only the outer surface layers. It is also possible to develop laboratory instrumentation which can acquire such data although at a greatly reduced rate. Instead of the typical one-hour running time used in the present study, several hours or overnight runs might be required. In this case, the advantage of preselecting the samples on the bas is of elemental composition is clearly indicated. 


\section{X-Ray Powder Diffractometry}

A second $x$-ray method for the chemical characterization of ambient aerosols is based on measurements of the unique crystallographic properties possessed by the solid particles. This well-known method of $x$-ray powder diffraction has long been used for the chemical identification of solid samples and its properties and procedures are well understood.

The method involves the measurement of the diffraction pattern produced by the sample when irradiated by an x-ray beam. A given crystal lattice plane will diffract an $x$-ray beam at a specific angle if the Bragg condition is satisfied. That is, $\lambda=2 d \sin \theta$, where $\lambda$ is the incident $x-r a y$ wavelength, $d$ is the lattice spacing and $\theta$ is the angle which the $x$-ray makes with the lattice plane. For polycrystalline samples, one can assume that there are always a few microcrystals that are at the proper orientation to satisfy this concition for a given $\lambda$ and $\theta$. The measurement then consists of recording the intensity of a mono-energetic diffracted beam as a function of diffraction angle. One then obtains a spectrum of peaks corresponding to reflections from the major lattice planes. Insofar as the lattice spacings and crystal symmetry are unique to a given compound, one can compare the values observed for an unknown specimen with those contained in extensive data compilations.

In order to adapt the techrique to the problem of aerosol samples deposited on filters, we have modified the normal diffractometer geometry to incorporate a position-sensitive detector in a manner similar to that of Gobe1[13]. Figure 5 is a schematic Jiagram of the apparatus. Normal diffractometers employ a line source and a slit image detector in order to precisely define the angle $\theta$ at which the diffraction occurs. This 
restrictive geometry results in a prohibitive? $\because$ ?ow counting rate for the case of dilute specimens. We have replaced the normal detector with a pressurized gas multiwire proportional chamber. The position-sensitive feature of the chamber acts as a multiple slit detector and results in a twenty-fold increase in counting rate over a single slit design. The spacial resolution of the present detector is $200 \mu \mathrm{m}$ over a linear distance of $1 \mathrm{~cm}$.

The measurement consists of rotating sample and detector using a standard diffractometer goniometer. The linear position of the eyent recorded in the detector is encoded in terms of an equivalent angle and the result added to the angular position of the rotating detector arm. By continuousiy ercoding data in this fashion, one averages over any non-uniformities in the detector resporse over its active length. Furthermore, because of the angular extension of the detector, it is possible to satisfy the Bragg condition for a given $\theta$ and $d$ over a range of orientations of the sample. This implies that the contributions to the diffraction peaks are averaged over a larger number of small crystals at yarious orientations. This averiging process reduces spectral artifacts resulting from particle size effects and sample inhomogeneity.

Figure 5 is a series of plots showing the diffraction spectra obtained using $\mathrm{C} u \mathrm{~K}_{\boldsymbol{a}}$ radiation on a coarse particle sample and similar spectra of standard mineral samples of calcite and quartz. The obvious overlap of major lines indicates that the unknown sample is a combination of the two compounds.

In a typical analytical program, one could maintain a file of standaró 
spectra of important compounds which could be compared to the unknown spectrum. Lines which are not accounted for could be referenced against the larger powder diffraction data files. It should be emphasized that the results of powder diffraction measurements are dominated by the major constituents of the sample and have a limited ability to detect and identify minor components in the specimen. Because of the complexity of the diffraction patterns exhibited by these mixtures, occasional ambiguities of interpretation can result particularly for the weaker components in the composite spectrum. Again, the availability of elemental concentrations has been a valuable tool for eliminating such ambiguities.

\section{Summary and Conclusions}

We have demonstrated the application of a series of $x$-ray methods to the airaiysis of air particulate samples. Although the present inethods are largeiy adapted from known procedures, the application of modern x-ray source and detector technology have greatly enhanced their capabilities. In particular, the analysis of the dilute specimens obtained from modern air samplers would not be practical without the improvements described. Many advantages of $x$-ray-based methods have been shown, including sensitivity, convenience and the non-destructive nature of the procedures. This latter feature allows the multiple analysis of the same sample by all of the above methods and subsequent verification by other methods if necessary. 


\section{Acknowledgements}

The authors would like to acknowledge the technical assistance of other members of the Department of Irstrument Science and Engineering particulariy Raymond Gatti and hilliam Searles.

This work was supported in part by the U.S. Environmental Protection Agency under interagency agreement number IAG AD-89-F-0-013-0 with the Assistant Secretary for Environment, Office of Health and Environmental Research, Poilutant Characterization and Safety Research Division of the U.S. Department of Energy under Contract No. W-7405-ENG-48 . 
Table 1. Detectable limits and sensitivity for energy dispersive $x$-ráy fluorescence analysis.

\begin{tabular}{|c|c|c|c|}
\hline ELEMENIT & $\begin{array}{l}\text { ATONIC } \\
\text { NUMBER }\end{array}$ & $\begin{array}{c}\text { MINIMUM } \\
\text { DETECTABLE } \\
\text { LIMIT } \\
\text { (ngm/sm²) }\end{array}$ & $\begin{array}{l}\text { SENSITIVITY } \\
\text { (counts } / \text { sec } \\
\text { per } \mu g \mathrm{~m} / \mathrm{cm}^{2} \text { ) }\end{array}$ \\
\hline $\begin{array}{l}\left.A I^{a}\right) \\
\mathrm{Si} \\
\mathrm{P} \\
\mathrm{S} \\
\mathrm{C} ? \\
\mathrm{~K} \\
\mathrm{Ca}\end{array}$ & $\begin{array}{l}13 \\
14 \\
15 \\
16 \\
17 \\
19 \\
20\end{array}$ & $\begin{array}{c}200.0 \\
58.9 \\
32.9 \\
29.4 \\
26.6 \\
9.14 \\
7.7\end{array}$ & $\begin{array}{c}7.40 \\
25.3 \\
48.0 \\
83.8 \\
125.0 \\
272.0 \\
411.0\end{array}$ \\
\hline $\begin{array}{l}\left.\mathrm{T}^{\mathrm{i}} \mathrm{b}\right) \\
\mathrm{Cr} \\
\mathrm{Mn} \\
\mathrm{Fe} \\
\mathrm{Ni} \\
\mathrm{Cu} \\
\mathrm{Zn} \\
\mathrm{Go} \\
\mathrm{As} \\
\mathrm{Se} \\
\mathrm{Br} \\
\mathrm{Rb} \\
\mathrm{Sr} \\
\mathrm{Hg} \\
\mathrm{Pb}\end{array}$ & $\begin{array}{l}22 \\
23 \\
24 \\
25 \\
26 \\
28 \\
29 \\
30 \\
31 \\
33 \\
34 \\
35 \\
37 \\
38 \\
80 \\
82\end{array}$ & $\begin{array}{r}31.3 \\
22.2 \\
16.6 \\
13.5 \\
11.8 \\
6.2 \\
6.5 \\
5.3 \\
3.8 \\
3.1 \\
2.6 \\
2.7 \\
2.8 \\
3.8 \\
6.0 \\
9.1\end{array}$ & $\begin{array}{r}28.8 \\
37.8 \\
49.3 \\
59.8 \\
76.4 \\
112.0 \\
128.0 \\
148.0 \\
166.0 \\
209.0 \\
234.0 \\
258.0 \\
304.0 \\
320.0 \\
109.0 \\
109.0\end{array}$ \\
\hline $\begin{array}{l}\mathrm{Cd} C) \\
\mathrm{Sn} \\
\mathrm{Sb} \\
\mathrm{Ba}\end{array}$ & $\begin{array}{l}48 \\
50 \\
51 \\
56\end{array}$ & $\begin{array}{r}5.9 \\
7.8 \\
8.1 \\
31.0\end{array}$ & $\begin{array}{l}75.5 \\
75.7 \\
74.6 \\
62.1\end{array}$ \\
\hline
\end{tabular}
a) Ti secondary target, 90-second analysis
b) Mo secondary $t$ arget, $80-$ second ana lysis
c) Sm secondary target, 150-second analys is 


\section{Figure Captions}

1) Experimental system used for $x$-ray absorption spectroscopy. The variable energy $x$-ray bean is derived from the synchrotron radiation using a crystal monochromator. In the formulae, $\tau(E)$ is the photoelectric cross section $\left(\mathrm{cm}^{2} / \mathrm{gm}\right), 0$ is the density and $x$ is the thickness.

2) Representative absorption spectra obtained at the $2 \mathrm{n} \mathrm{K}$-shell binding energy for a series of standard compounds of potential interest in urban aerosol studies.

3) Spectra obtained for the $2 n$ K-absorption edge from two samples acquired simultaneously at an urban sampling site.

4) Schematic diagram of $x$-ray pcisder diffraction system using a multiwire proportional chamber.

5) X-ray powder diffraction paiterns for a coarse-particie air particulate sample togetner with two standard materials.

\section{References}

[ 1] T.G. Dzubay, ed., X-Ray Fluorescence Analys is of Environmental Samples Ann Arbor Science, Ann Arbor (1977).

[ 2] G.S. Kowalczyk, C.E. Choquette and G.E. Gordon, Atmos. Environ. 12 (1978) 1143.

[ 3] B.W. Loo, J.M. Jak?evic end F.S. Goulding Fine Partic les: Aerosol Generation, Sampling and Analys is, B.Y.H. Liu, ed., Academic Press, New York (1976) 311 .

[ 4] T.G. Dzubay and R.K. Stevens, Environ. Sci. Technol. 9 (1975) o63.

[ 5] J.M. Jaklevic, R.C. Gatti, F.S. Goulding, B.W. Loo and A. Thompson, Proceedings 4th Joint Conference on Sensing of Environmental Pollutants, New Orleans, LA, (Nov. 1977).

[6] J.M. Jaklevic, R.C. Gatti, F.S. Goulding, B.W. Loo and A.C. Thompson, Environmental Protection Agency Report No. EPA-600/54-81-006 (Mar. 1981).

[ 7] S.K. Friedlander, Enviran. Sci. Technol. 7 (1973) 235. 
[ 8] G.E. Gordon, Environ. Sci. Technol, 14 (1980) 792.

[ 9] P.K. Hopke, E.S. Gladney, G.E. Gordon, W.H. Zoller and A.G. Jones, Atmos. Environ. 10 (1976) 1015.

[10] A.J. Ramponi ario J.M. Jaklevic, Lawrence Berkeley Laboratory Report No. LBL-7284, UC-37 (Dec. 1978).

[11] J.M. Jaklevic, J.A. Kirby, A.J. Ramponi and A.C. Thompson, Environ. Sci. Technol. 14 (1980) 437.

[12] H. Winick and S. Doniack, eds., Synchrotron Radiation Research Plenum Press, N.Y. (1980).

[13] H.E. Gobel, Adv. X-Ray Anal. 22 (1979) 247. 


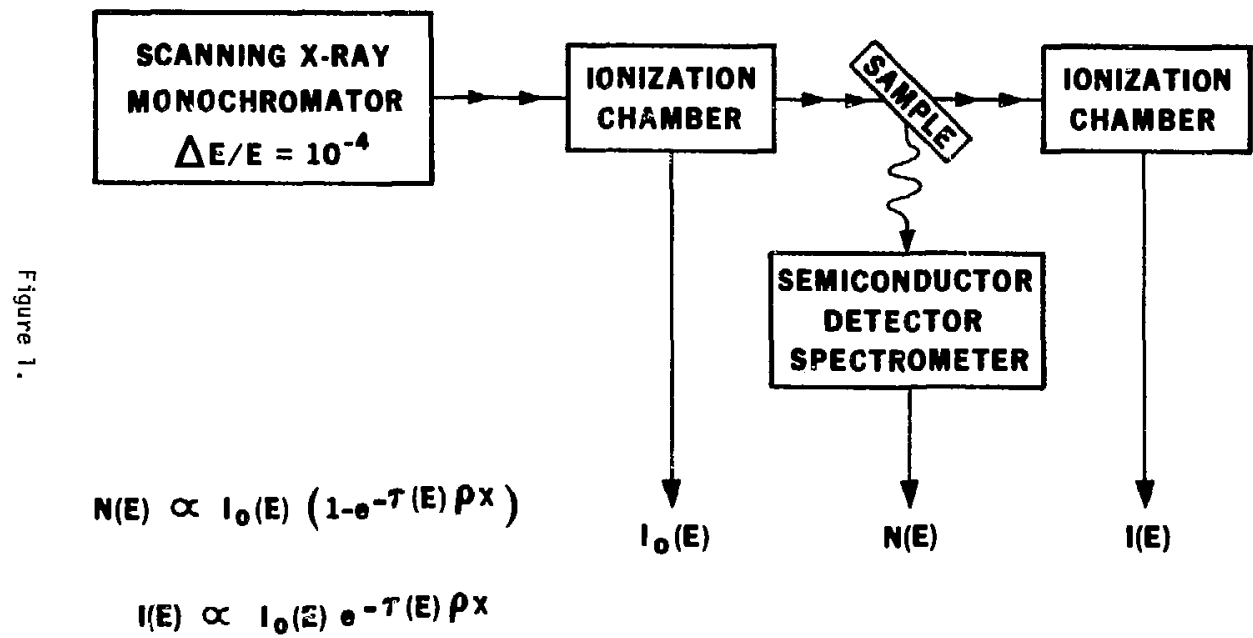


$-16-$

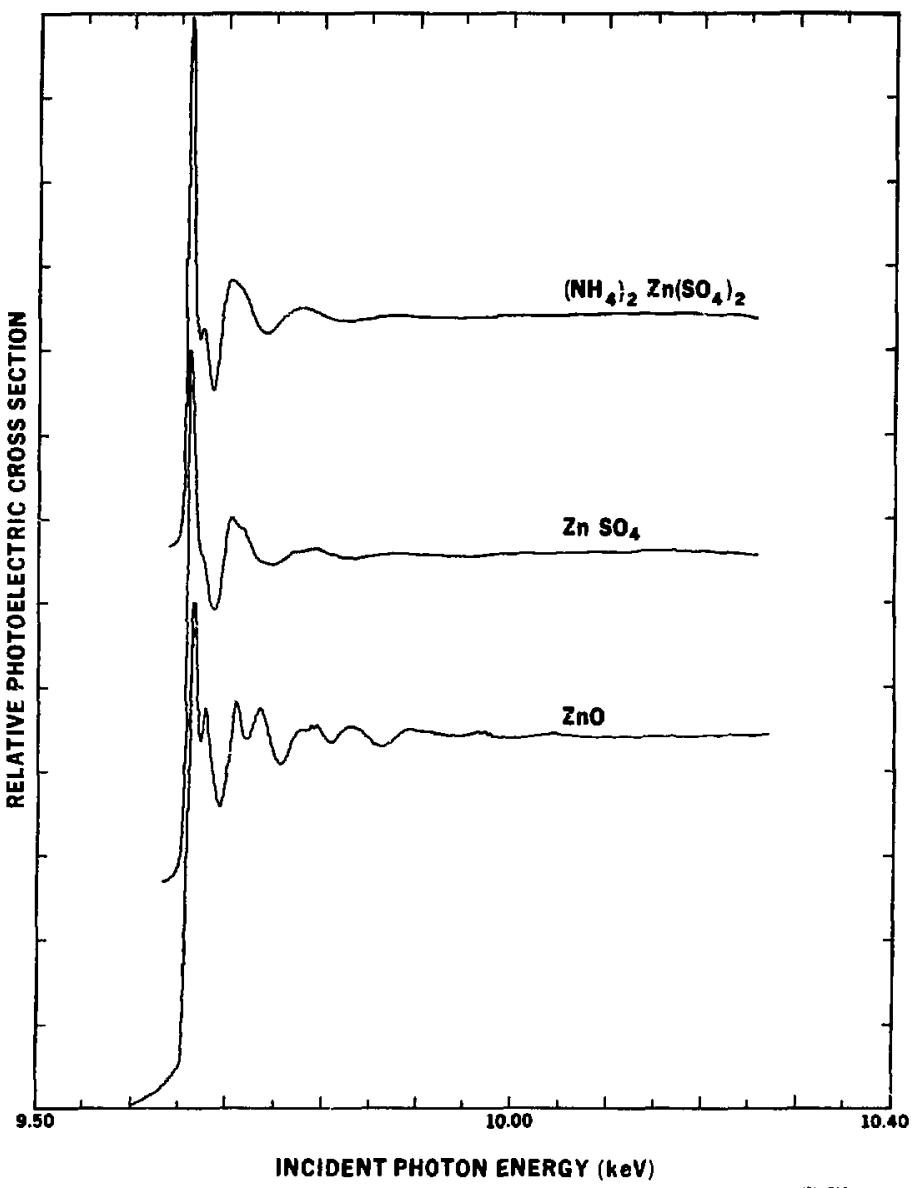

Figure 2.

xBL $747-10509$ 


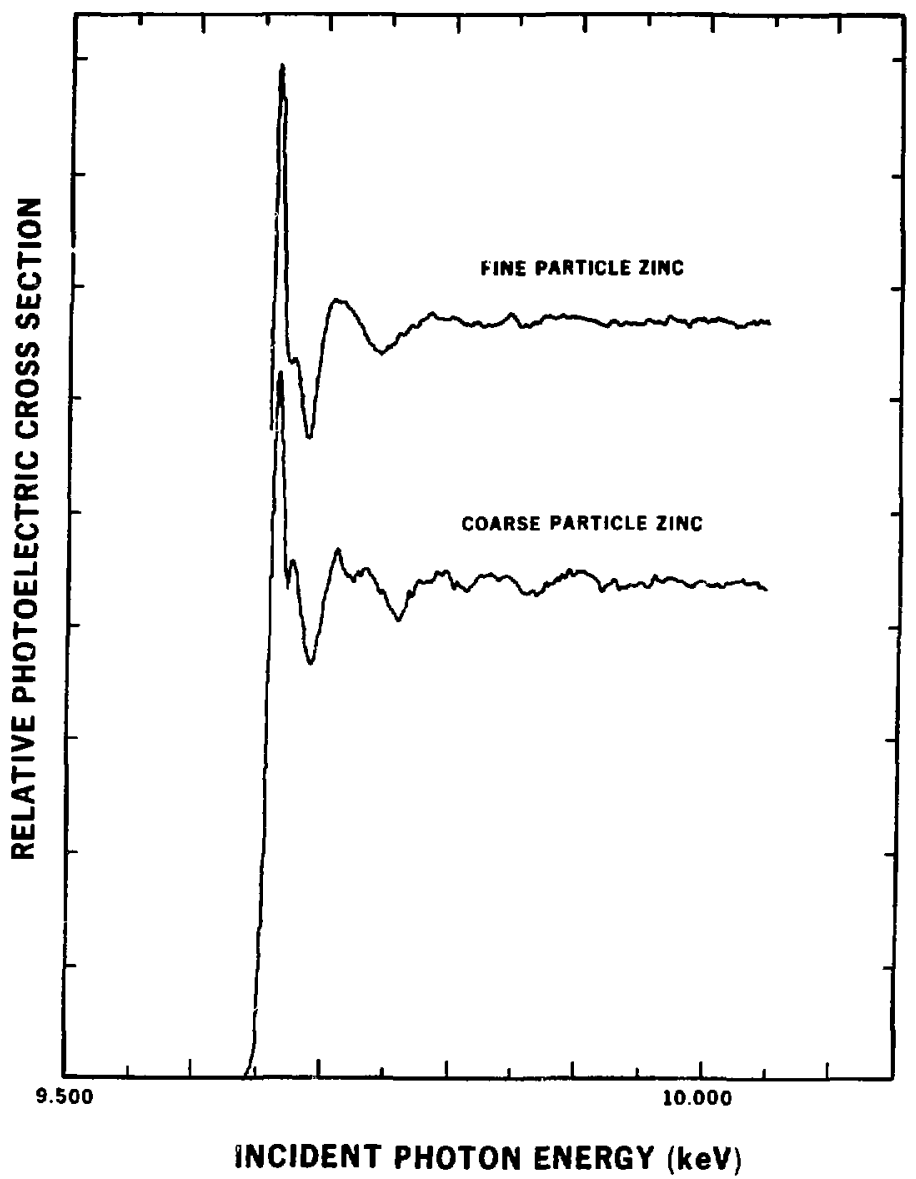

Figure 3. 


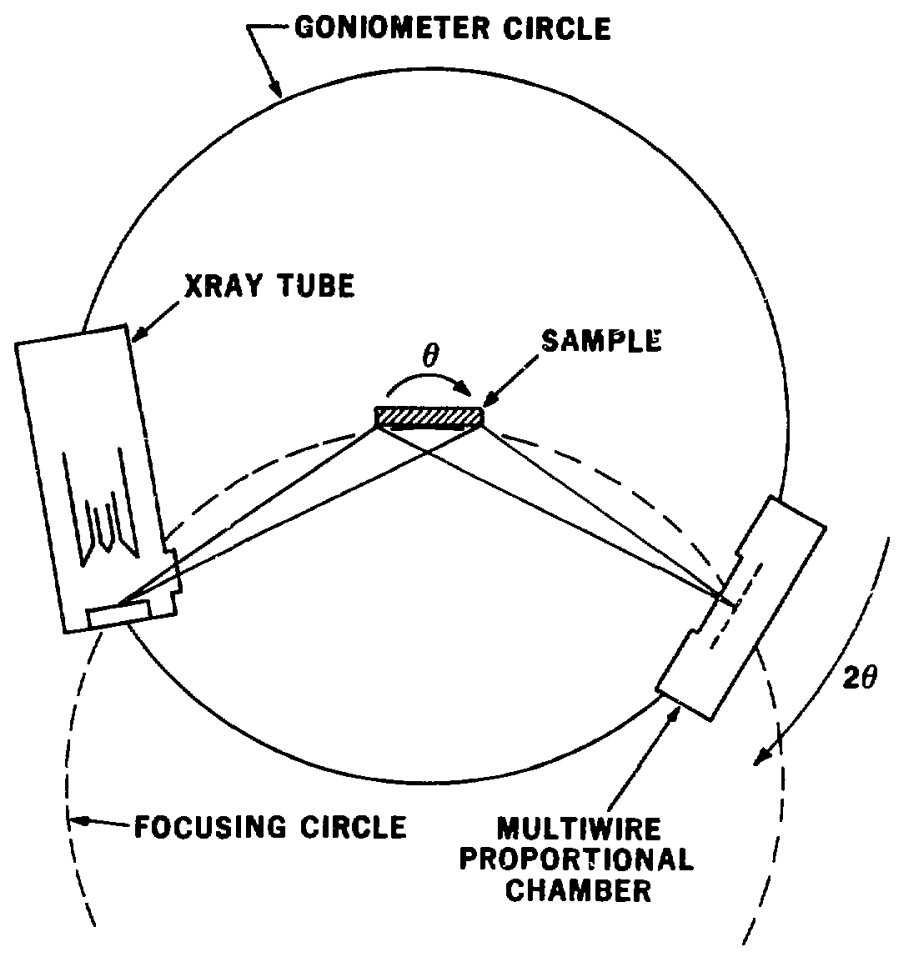

XBL 815-9619

Figure 4. 


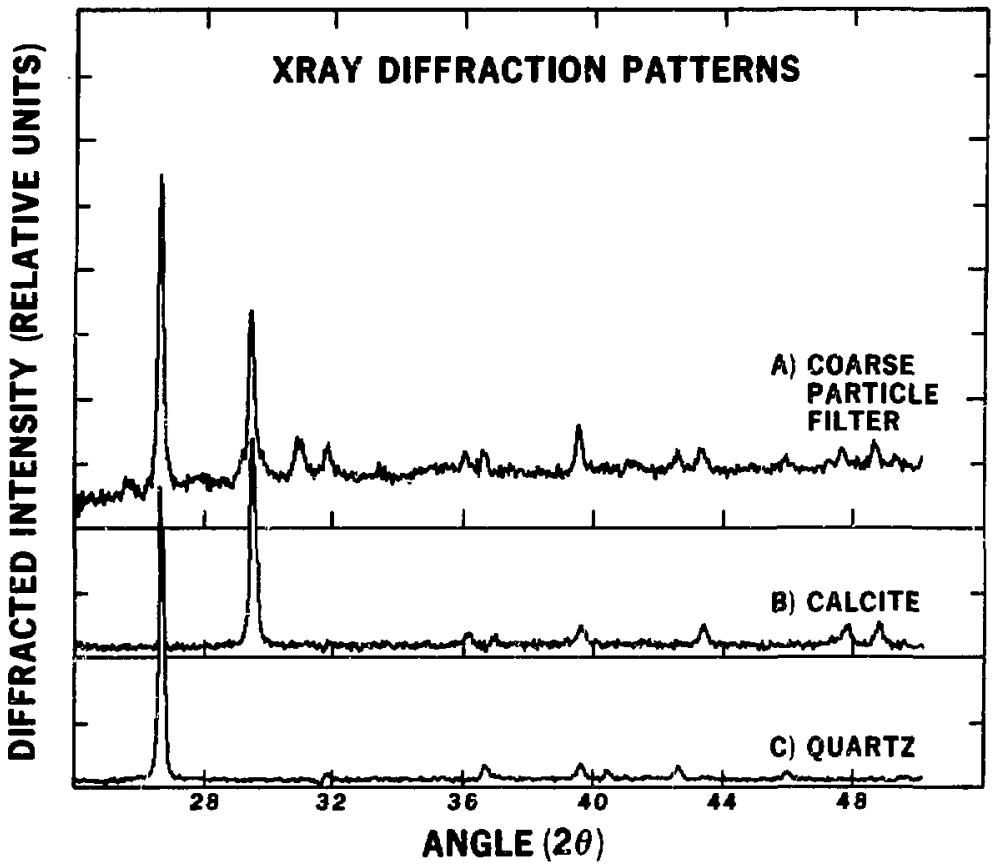

XBL 815-9618

Figure 5. 